OPEN ACCESS

Edited by:

Guowei Li,

Guangdong Second Provincial

General Hospital, China

Reviewed by:

Yutao Guo,

Chinese PLA General Hospital, China Lehana Thabane,

McMaster University, Canada

${ }^{*}$ Correspondence:

Yumei Xue

xymgdci@163.com

${ }^{\dagger}$ These authors have contributed equally to this work

Specialty section

This article was submitted to Family Medicine and Primary Care,

a section of the journal

Frontiers in Medicine

Received: 03 January 2020

Accepted: 23 April 2020

Published: 04 June 2020

Citation:

Tang L, Liu H, Deng H, Zhan X,

Fang X, Liao H, LiU Y, Fu L, Fu Z,

Liu H, Wu S and Xue Y (2020)

Minimally Interrupted Non-Vitamin $\mathrm{K}$ Antagonist Oral Anticoagulants vs. Bridging Therapy and Uninterrupted Vitamin K Antagonists During Atrial Fibrillation Ablation: A Retrospective

Single-Center Study.

Front. Med. 7:197.

doi: 10.3389/fmed.2020.00197

\section{Minimally Interrupted Non-Vitamin K Antagonist Oral Anticoagulants vs. Bridging Therapy and Uninterrupted Vitamin K Antagonists During Atrial Fibrillation Ablation: A Retrospective Single-Center Study}

\author{
Lihong Tang ${ }^{1,2+}$, Haiyan Liu ${ }^{1,2 t}$, Hai Deng 1,2, Xianzhang Zhan 1,2, Xianhong Fang 1,2, \\ Hongtao Liao ${ }^{1,2}$, Yang Liu ${ }^{1,2}$, Lu Fu ${ }^{1,2}$, Zuyi Fu ${ }^{1,2}$, Huiyi Liu ${ }^{1,2}$, Shulin $\mathrm{Wu}^{1,2}$ and \\ Yumei Xue ${ }^{1,2 *}$
}

${ }^{1}$ Guangdong Cardiovascular Institute, Guangdong Provincial People's Hospital, Guangdong Academy of Medical Sciences, Guangzhou, China, ${ }^{2}$ Guangdong Provincial Key Laboratory of Clinical Pharmacology, Guangdong Provincial People's Hospital, Guangdong Academy of Medical Sciences, Guangzhou, China

Objectives: Although the latest international guidelines recommend the use of uninterrupted non-vitamin K antagonist oral anticoagulants (NOAC) during atrial fibrillation (AF) ablation, it does not reflect current clinical practice, as most centers still use a minimally interrupted NOAC strategy. The purpose of this study was to evaluate the safety and effectiveness of minimally interrupted NOAC compared with bridging therapy and uninterrupted vitamin $\mathrm{K}$ antagonist (VKA) for nonvalvular $\mathrm{AF}$ ablation.

Patients and Methods: A total of 4520 patients who underwent AF ablation between January 2010 and December 2018 were included in the analysis. According to their periprocedural anticoagulation strategies, patients were divided into three groups: Bridging heparin group ( $n=1848)$; Uninterrupted VKA group $(n=796)$ and Minimally interrupted NOAC group (Total $n=1876$; dabigatran: $n=865$; rivaroxaban, $n=1011$ ). A combined complication endpoint (CCE) as composed of any bleeding complications and thromboembolic events was analyzed.

Results: Rates of thromboembolisms were similar among the three groups $(0.22 \%$ for Bridging heparin group, $0.25 \%$ for Uninterrupted VKA group, and $0.11 \%$ for Minimally interrupted NOAC group, $p=0.626)$. There was a significant difference among the three groups for the incidence of overall bleeding events (8.50\% for Bridging heparin group, $4.52 \%$ for Uninterrupted VKA group, and $2.67 \%$ for Minimally interrupted NOAC group, $p<0.001$ ). A significant difference of CCE rates was shown in the Minimally interrupted NOAC group as compared with the Uninterrupted VKA group (2.77 vs. $4.77 \%, p=0.008$ ) and the Bridging heparin group (2.77 vs. $8.71 \%, p<0.001$ ). There was no significant difference in CCE rates among the different NOACs (dabigatran $2.89 \%$ vs. rivaroxaban $2.67 \%, p=0.773)$. 
Conclusions: In patients undergoing AF ablation, minimally interrupted NOACs during the periprocedural period appears safer and equally effective when compared to the bridging heparin and uninterrupted VKA therapy.

Keywords: ablation, anticoagulation, atrial fibrillation, bleeding events, thromboembolism

\section{INTRODUCTION}

Catheter ablation is a safe, effective, and promising strategy for patients with symptomatic atrial fibrillation (AF). Careful attention to periprocedural anticoagulation for AF ablation is mandatory to reduce the risk of major complications comprising stroke, transient ischemic attack (TIA), and cardiac tamponade (1). Currently, it is a well-established anticoagulation strategy that uninterrupted warfarin therapy is associated with a lower risk of periprocedural bleeding and strokes than stopping vitamin $\mathrm{K}$ antagonist (VKA) and bridging with heparin (2). However, in an era in which non-vitamin $\mathrm{K}$ antagonist oral anticoagulants (NOACs) are increasingly used, recent randomized controlled trials (RCTs) have demonstrated that uninterrupted NOAC is as safe and effective in comparison to uninterrupted VKA in patients undergoing AF ablation (3-6). The 2017 Consensus Statement on Catheter and Surgical Ablation of AF give a class I or IIa indication to perform AF ablation without withholding the NOACs and VKAs or withholding one to two doses of the NOACs prior to ablation (1).

Recent meta-analysis reported a similar incidence of thromboembolic events and lower incidence of bleeding in patients with either interrupted or uninterrupted NOACs therapy as compared with uninterrupted VKAs therapy (7-9). However, the major concern in performing AF ablation with uninterrupted NOACs is the risk of bleeding, particularly lifethreatening bleeding such as pericardial tamponade. At present, the uninterrupted NOAC strategy does not reflect current clinical practice, as most centers still use a minimally interrupted NOAC strategy (10). The aim of the present study was to evaluate the safety and effectiveness of minimally interrupted NOAC compared with bridging therapy and uninterrupted VKA during AF ablation in a "real-world" clinical practice.

\section{PATIENTS AND METHODS}

\section{Study Population}

Consecutive patients who underwent catheter ablation of $\mathrm{AF}$ from January 2010 to December 2018 were recorded. The inclusion criteria were as follows: (1) adult patients ( $\geq 18$ years of age); (2) patients undergoing either radiofrequency ablation or cryoablation for AF. The exclusion criteria were as follows: (1) presence of valvular heart disease; (2) diagnosis of malignant tumors; and (3) incomplete follow-up data within 30 days postablation.

Depending on the periprocedural anticoagulation strategies prescribed by prior physicians, patients were divided into three groups. The first group included patients who discontinued warfarin and bridging with heparin. The second group included patients who used periprocedural uninterrupted VKA. The third group included patients who used periprocedural minimally interrupted NOAC (Dabigatran: $110 \mathrm{mg}$ twice daily; Rivaroxaban: 15 or $10 \mathrm{mg}$ once daily). A transesophageal echocardiography (TEE) was routinely performed on the same day or 1 day before ablation to exclude left atrial appendage (LAA) thrombus. Computed tomography (CT) scans were performed only in a minority of patients who were intolerant to TEE.

The studies involving human participants were reviewed and approved by the Clinical Research Ethics Committee of Guangdong Provincial People's Hospital. The patients provided their written informed consent to participate in this study.

\section{Periprocedural Protocol}

In the Bridging heparin group, warfarin was usually withheld for 3-5 days, and enoxaparin $1 \mathrm{mg} / \mathrm{kg}$ every $12 \mathrm{~h}$ was given until the evening before the ablation procedure with a target international normalized ratio $(\mathrm{INR})<2$. In the Uninterrupted VKA group, with patients using continued warfarin, the target INR level at the day of the procedure was 2-3. In the Minimally interrupted NOAC group, the last dabigatran was given in the evening on the day before the procedure, while rivaroxaban was given on the morning of the day before the procedure. On the morning of the procedure NOACs were paused. Neither low molecular weight heparin (LMWH) nor unfractionated heparin was administered during the preprocedural period in both the Uninterrupted VKA group and Minimally interrupted NOAC group.

During the procedure, intravenous heparin $(100 \mathrm{U} / \mathrm{kg})$ was given immediately after transseptal puncture and adjusted to maintain a target activated clotting time (ACT) of 250-350 s. Protamine was not routinely used at the end of the procedure.

As for the Bridging heparin group, enoxaparin $0.5 \mathrm{mg} / \mathrm{kg}$ twice daily was used as a bridge to resumption of warfarin therapy until INR $\geq 2$. In patients treated with uninterrupted VKA who had an INR 2.0 or greater on the day of the procedure, warfarin was continued the evening of the procedure with a target INR level of 2-3. In patients treated with minimally interrupted NOAC preablation dabigatran or rivaroxaban was restarted $3-5 \mathrm{~h}$ after ablation. No LMWH or unfractionated heparin was administered in both the Uninterrupted VKA group and Minimally interrupted NOAC group after ablation. All patients were continued on anticoagulation for at least 2 months postablation.

\section{Definitions}

Any bleeding or thromboembolic event during the procedure and within 30 days postablation was categorized. Bleeding complications were defined by the Bleeding Academic Research 


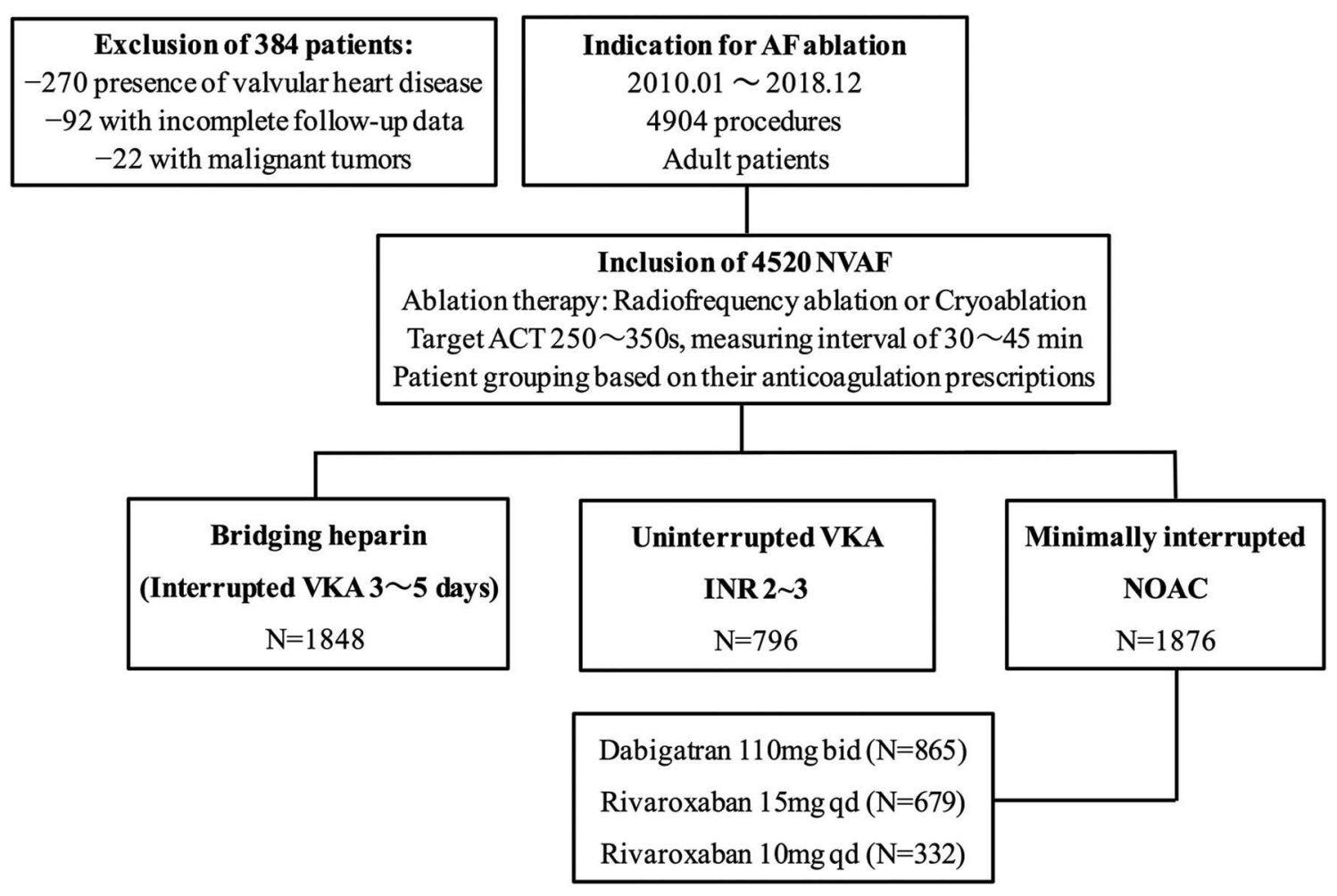

FIGURE 1 | Study flow chart. AF, atrial fibrillation; NVAF, nonvalvular atrial fibrillation; ACT, activated clotting time; VKA, vitamin K antagonist; NOAC, non-vitamin K antagonist oral anticoagulant.

Consortium (BARC) and International Society on Thrombosis and Haemostasis (ISTH) $(11,12)$. Accordingly, major bleeding events included cardiac tamponade or pericardial effusions requiring drainage, intracranial and major gastrointestinal hemorrhages, a hemothorax, retroperitoneal bleeding, any bleeding requiring a blood transfusion, and vascular access site complications requiring any intervention. Bleeding events that did not fulfill the ISTH criteria for major bleeding were considered nonmajor bleeding events. Thromboembolic events were defined as the occurrence of symptomatic stroke/transient ischemic attack (TIA), peripheral embolic events, or deep venous thrombosis. However, no routine postablation brain magnetic resonance imaging (MRI) was performed to test for "silent" cerebral events. Furthermore, we defined a combined complication endpoint (CCE) as composed of any bleeding complications and thromboembolic events.

\section{Statistical Analysis}

Continuous variables were presented as mean $\pm \mathrm{SD}$ and compared using one-way analysis of variance (ANOVA) test. Categorical variables were presented as counts and percentages and compared using the $\chi^{2}$ test or Fisher exact test as appropriate. Multivariable logistic regression was used to identify significant predictors of CCE. All potential confounders were entered into the model on the basis of known clinical relevance or significant association observed in univariate analysis.
The adjusted odds ratio and $95 \%$ confidence interval (CI) were computed.

Four confounders (age, type of $\mathrm{AF}, \mathrm{CHA}_{2} \mathrm{DS}_{2}$-VASc score, and HAS-BLED score) were adjusted for unequal patient characteristics due to nonrandomization. After that, a KaplanMeier analysis and log-rank test were used to compare the CCE rates within 30 days after ablation procedure among groups. A $p$ value $<0.05$ was considered statistically significant. All statistical analysis was performed using SPSS software (version 24.0, IBM, Armonk, NY, USA).

\section{RESULTS}

Of a total of 4,904 patients who underwent AF ablation, 384 were excluded due to the coexistence of valvular heart disease, malignant tumors, and incomplete follow-up data within 30 days postablation. Among the remaining 4520 patients, AF ablation was performed with a "Bridging heparin" protocol in 1848 patients $(40.9 \%)$, an "Uninterrupted VKA" protocol in 796 patients (17.6\%), and a "Minimally interrupted NOACs" protocol in 1876 patients (41.5\%) (Figure 1). The majority of patients were male $(65.9 \%)$, and the average age was 58 years. The median $\mathrm{CHA}_{2} \mathrm{DS}_{2}$-VASc score was 1 , and a $\mathrm{CHA}_{2} \mathrm{DS}_{2}$ VASc $\geq 2$ accounted for $43.3 \%$ of the cohort with no significant difference among the three groups $(p=0.255)$. Patients had a median HAS-BLED of 1 (range $0-4$ ), with the majority being 
TABLE 1 | Baseline characteristics.

\begin{tabular}{|c|c|c|c|c|}
\hline & $\begin{array}{l}\text { Bridging heparin } \\
\qquad(N=1848)\end{array}$ & $\begin{array}{l}\text { Uninterrupted VKA } \\
\qquad(N=796)\end{array}$ & $\begin{array}{l}\text { Minimally interrupted NOAC } \\
\qquad(N=1876)\end{array}$ & $p$-value \\
\hline Age, years & $57 \pm 11$ & $60 \pm 11$ & $59 \pm 11$ & $<0.001$ \\
\hline Male, $n(\%)$ & $1227(66.4)$ & $511(64.2)$ & 1239 (66.0) & 0.537 \\
\hline Persistent AF, $n$ (\%) & $386(20.9)$ & $167(21.0)$ & $377(20.1)$ & 0.753 \\
\hline Hypertension, n (\%) & $758(41.0)$ & $310(38.9)$ & $731(39.0)$ & 0.381 \\
\hline Diabetes mellitus, n (\%) & $234(12.7)$ & $109(13.7)$ & $227(12.1)$ & 0.523 \\
\hline CAD, $n(\%)$ & $272(14.7)$ & $118(14.8)$ & 309 (16.5) & 0.288 \\
\hline Vascular disease, $n$ (\%) & $203(11.0)$ & $73(9.2)$ & $231(12.3)$ & 0.058 \\
\hline Heart failure, $n(\%)$ & $50(2.7)$ & $25(3.1)$ & $55(2.9)$ & 0.814 \\
\hline Prior stroke/TIA, $n$ (\%) & $114(6.2)$ & $52(6.5)$ & $124(6.6)$ & 0.851 \\
\hline Renal dysfunction, $n$ (\%) & $38(2.1)$ & $23(2.9)$ & $27(1.4)$ & 0.042 \\
\hline $\mathrm{CHA}_{2} \mathrm{DS}_{2}-\mathrm{VASc} \geq 2, n(\%)$ & $776(42.0)$ & $360(45.2)$ & $823(43.9)$ & 0.255 \\
\hline HAS-BLED $\geq 3, n(\%)$ & $33(1.8)$ & $26(3.3)$ & $62(3.3)$ & 0.009 \\
\hline
\end{tabular}

Values are given as the mean $\pm S D$ or $n(\%)$.

$C A D$, coronary artery disease; $C H A_{2} D S_{2}$-VASc score, congestive heart failure, hypertension, diabetes mellitus, vascular disease, an age of 65-74 years, female sex (1 point for each), age $\geq 75$ years, stroke/TIA (2 points for each); HAS-BLED, hypertension, abnormal renal and liver function, a stroke, bleeding history/predisposition, labile international normalized ratio, age > 65 years, drugs/alcohol concomitantly (1 point for each); NOAC, non-vitamin K antagonist oral anticoagulant; TIA, transient ischemic attack; VKA, vitamin K antagonist.

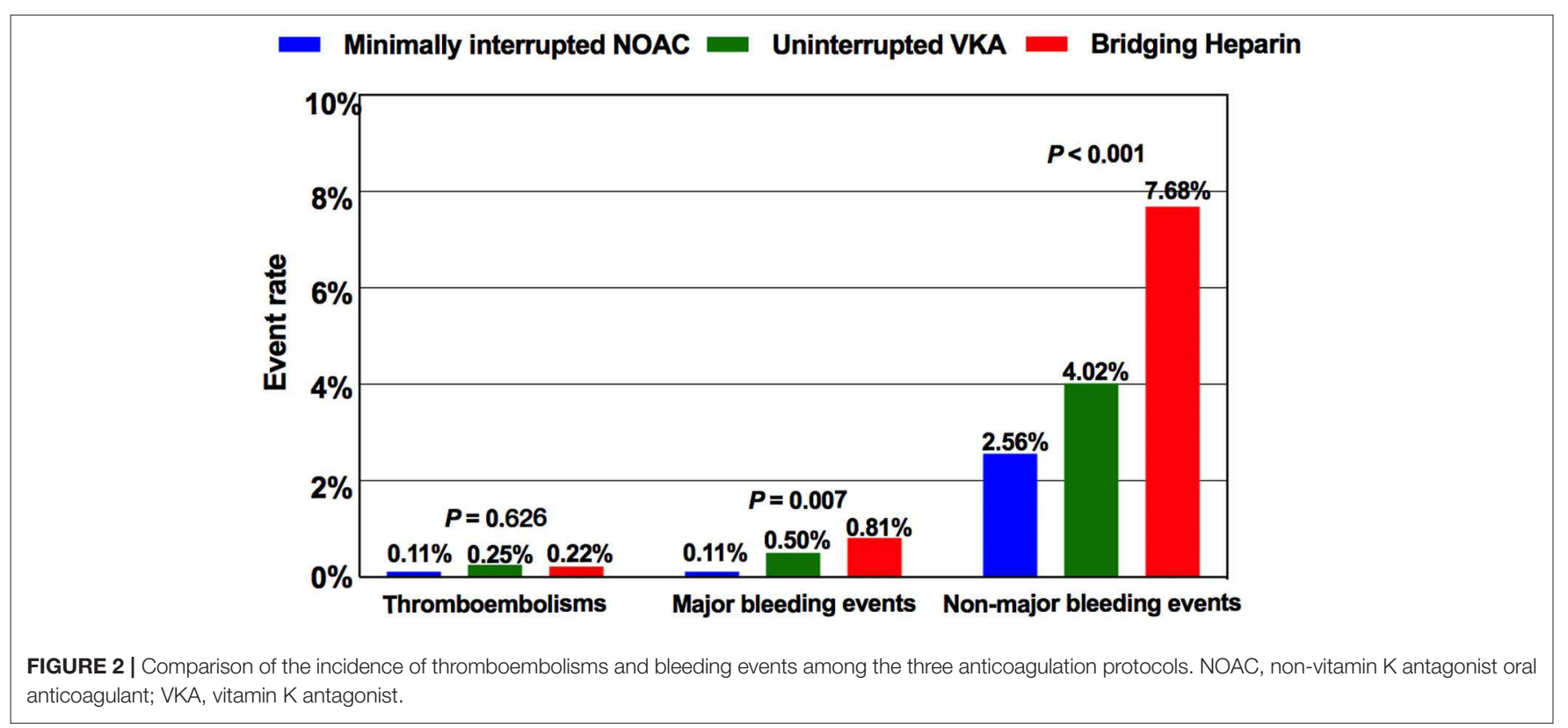

classed as 0 or 1 across groups and $2.7 \%$ having a score $\geq 3$. There were significant differences among groups with respect to their bleeding risk $(p=0.009)$. Baseline characteristics of the three groups are summarized in Table 1. Furthermore, the incidence of thromboembolisms and bleeding events among the three anticoagulation strategies are shown in Figure 2.

\section{Bleeding Complications}

In our study, 243 overall bleeding events were reported; the overall event rate was $8.50 \%$ in patients receiving bridging regimen with $\mathrm{LMWH}, 4.52 \%$ in patients receiving uninterrupted warfarin, and $2.67 \%$ in patients receiving minimally interrupted NOACs (Table 2). There was significant difference among the three groups for the incidence of overall bleeding events ( $p$ $<0.001)$. The number of major bleeding events was similar between the Minimally interrupted NOACs group and the Uninterrupted warfarin group ( 0.11 vs. $0.50 \%$, OR $0.21 ; 95 \%$ CI $0.04-1.16 ; p=0.073$ ), while a lower trend was observed for the comparison between the Minimally interrupted NOACs group and the Bridging Heparin group (0.11 vs. $0.81 \%$, OR 0.36; $95 \%$ CI $0.17-0.76 ; p=0.007)$.

In a further breakdown of major bleeding, there were a total of 21 pericardial tamponades requiring drainage (total incidence $0.46 \%$; 15 in the Bridging heparin group, 4 in the Warfarin group, and 2 in the NOAC group, Table 3). Among them, a majority of the events $(17 / 21,81.0 \%)$ occurred during the procedure, 
TABLE 2 | Details on bleeding and thromboembolic complication rates.

\begin{tabular}{|c|c|c|c|}
\hline & $\begin{array}{l}\text { Bridging } \\
\text { heparin }\end{array}$ & $\begin{array}{l}\text { Uninterrupted } \\
\text { VKA }\end{array}$ & $\begin{array}{l}\text { Minimally } \\
\text { interrupted } \\
\text { NOAC }\end{array}$ \\
\hline \multicolumn{4}{|l|}{ Major bleedings } \\
\hline Pericardial tamponade & $15(0.81)$ & $4(0.50)$ & $2(0.11)$ \\
\hline Other major bleedings ${ }^{a}$ & 0 & 0 & 0 \\
\hline Total & $15(0.81)$ & $4(0.50)$ & $2(0.11)$ \\
\hline \multicolumn{4}{|l|}{ Non-major bleedings } \\
\hline $\begin{array}{l}\text { Pericardial effusion not } \\
\text { requiring drainage }\end{array}$ & $5(0.27)$ & 0 & $1(0.05)$ \\
\hline Groin bleeding/hematoma & $72(3.90)$ & 19 (2.39) & $26(1.39)$ \\
\hline Femoral pseudoaneurysm & $5(0.27)$ & $1(0.13)$ & 0 \\
\hline Femoral arteriovenous fistula & 0 & $1(0.13)$ & $3(0.16)$ \\
\hline Other non-major bleedings ${ }^{b}$ & $60(3.25)$ & $11(1.38)$ & $18(0.96)$ \\
\hline Total & $142(7.68)$ & $32(4.02)$ & $48(2.56)$ \\
\hline \multicolumn{4}{|l|}{ Thromboembolic complications } \\
\hline Stroke & $3(0.16)$ & $2(0.25)$ & $2(0.11)$ \\
\hline Deep venous thrombosis & $1(0.05)$ & 0 & 0 \\
\hline Total & $4(0.22)$ & $2(0.25)$ & $2(0.11)$ \\
\hline Combined bleedings total & $157(8.50)$ & $36(4.52)$ & $50(2.67)$ \\
\hline Combined complication endpoint & $161(8.71)$ & $38(4.77)$ & $52(2.77)$ \\
\hline
\end{tabular}

Values are presented as $n(\%)$.

NOAC, non-vitamin K antagonist oral anticoagulant; VKA, vitamin K antagonist.

${ }^{a}$ Other major bleedings including any bleeding event that required a blood transfusion and/or intervention.

${ }^{b}$ Other non-major bleedings including hematuria, epistaxis, ecchymosis, and minor gastrointestinal bleeding.

while others occurred within 6 hours after the procedure. Emergency percutaneous pericardiocentesis with subxyphoid access was performed in all 21 patients. The mean drained blood volumes were $686 \mathrm{~mL}$. Protamine was administered in 14 patients for periprocedural heparin reversal. Direct autologous blood transfusion (AutoBT) was performed routinely as salvage therapy to allow maintenance the hemodynamic status (13). Despite pericardiocentesis, anticoagulation reversal, and AutoBT, an emergent open-chest surgery was required in $11(52.4 \%)$ patients due to persistent reaccumulation of blood in the pericardial space. Most of the patients completely recovered without any deficit while one patient died of cardiorespiratory arrest without surgery. In addition, no other major bleeding events were recorded.

Non-major bleeding complications were the most frequent bleeding complications overall, led by minor groin complications $(127 / 243 ; 52.3 \%)$ not requiring any intervention treatment. Most of the minor groin complications (including groin bleeding/hematoma, arteriovenous fistula, and pseudoaneurysm) occurred in the first 3 days after ablation procedure. In addition to the vascular access complications mentioned earlier, the occurrence times of other non-major bleeding events were irregular. Patients in the Minimally interrupted NOACs group had a lower risk of suffering non-major bleeding events in comparison to both the Uninterrupted warfarin group (OR 0.63; 95\% CI $0.40-0.99 ; p=0.044$ ) and the Bridging heparin group
TABLE 3 | Clinical details of the pericardial tamponade.

\begin{tabular}{lc}
\hline Pericardial tamponade $(\boldsymbol{N}=\mathbf{2 1})$ & \\
\hline Female, $n$ (\%) & $6(28.6)$ \\
Age (years), mean \pm SD & $66 \pm 8$ \\
Anticoagulation strategy, $n$ (\%) & \\
$\quad$ Bridging heparin & $15(71.4)$ \\
Uninterrupted warfarin & $4(19.0)$ \\
$\quad$ Minimally interrupted NOACs & $2(9.5)$ \\
Ablation techniques, $n$ (\%) & \\
RFCA & $19(90.5)$ \\
Cryo & $2(9.5)$ \\
Time of event onset, $n$ (\%) & \\
Intraprocedure & $17(81.0)$ \\
Within $6 \mathrm{~h}$ postprocedure & $4(19.0)$ \\
Pericardiocentesis, $n$ (\%) & $21(100)$ \\
Drained blood volume (mL), mean \pm SD & $686 \pm 463$ \\
AutoBT, $n$ (\%) & $15(71.4)$ \\
Protamine used, $n$ (\%) & $14(66.7)$ \\
Surgey, $n$ (\%) & $11(52.4)$ \\
Death, $n$ (\%) & $1(4.8)$ \\
&
\end{tabular}

Values are given as the mean $\pm S D$ or $n(\%)$.

AutoBT, autologous blood transfusion; Cryo, cryoablation; NOAC, non-vitamin $K$ antagonist oral anticoagulant; $R F C A$, radiofrequency ablation.

(OR 0.56; 95\% CI 0.48-0.66; $p<0.001$ ). More details about non-major bleeding events are shown in Table 2.

\section{Thromboembolic Complications}

The incidence of thromboembolic events was low (Bridging heparin, 0.22\%; Uninterrupted VKA, 0.25\%; Minimally interrupted NOAC, $0.11 \%$ ) and not significantly different among groups $(p=0.626)$. Of the 8 thromboembolic complications documented, 7 (87.5\%; 2 in the Bridging heparin group, 3 in Uninterrupted VKA group, and 2 in the Minimally interrupted NOAC group) were ischemic stroke events. Clinical details of the 7 patients with stroke events are shown in Table 4. Most complications $(5 / 7,71.4 \%)$ occurred either during the procedure or within 1 day postablation. At the time of stroke events, more than one half of the patients were in sinus rhythm. Continuous anticoagulation, receiving catheter ablation, and/or left atrial appendage closure might not guarantee that the stroke events would be avoided. CT or MRI scan revealed lesions were usually located in the cerebellar hemisphere, basal ganglia, temporal lobe, and right thalamus. All patients received conservative treatments except one patient who was treated by using emergent stent retriever thrombectomy $2 \mathrm{~h}$ after stoke onset. Furthermore, one patient in the Bridging heparin group experienced deep venous thrombosis 5 days after the procedure. Finally, he had an uneventful recovery after conservative treatments.

\section{Combined Complication Endpoint and Subgroup Analysis}

Patients were divided into two groups according to CCE outcome status, and univariate analysis was performed to compare 
TABLE 4 | Patients, characteristics, and management of stroke complications.

\begin{tabular}{|c|c|c|c|c|c|c|c|c|}
\hline Case & $\begin{array}{l}\text { Age/ } \\
\text { sex }\end{array}$ & $\mathrm{CHA}_{2} \mathrm{DS}_{2}-\mathrm{VAS}_{\mathrm{C}}$ & Anticoagulant & Ablation & Time $^{a}$ & Rhythm ${ }^{a}$ & Lesion location & Management \\
\hline 1 & $61 / F$ & 3 & Bridge & RFCA & Intraoperative & SR & $\begin{array}{l}\text { Right cerebellar } \\
\text { hemisphere }\end{array}$ & Conservative treatments \\
\hline 2 & $71 / \mathrm{F}$ & 6 & Bridge & RFCA & Intraoperative & SR & Left basal ganglia & CAG and thrombectomy \\
\hline 3 & $74 / \mathrm{M}$ & 5 & Bridge & RFCA & $1 \mathrm{~d}$ postoperative & $\mathrm{AF}$ & $\begin{array}{l}\text { Left temporal lobe } \\
\text { and cerebellar } \\
\text { hemisphere }\end{array}$ & Conservative treatments \\
\hline 5 & 70/M & 1 & Uninterrupted warfarin & RFCA & 7 d postoperative & SR & $\begin{array}{l}\text { Right basal ganglia } \\
\text { and temporal lobe }\end{array}$ & CAG and conservative treatments \\
\hline 6 & $69 / M$ & 3 & NOAC (rivaroxaban) & Cryo+LAAC & $1 \mathrm{~m}$ postoperative & $A F$ & Right basal ganglia & Conservative treatments \\
\hline 7 & $48 / F$ & 1 & NOAC (dabigatran) & RFCA & 1 d postoperative & SR & $\begin{array}{l}\text { Left frontal lobe } \\
\text { and temporal lobe }\end{array}$ & Conservative treatments \\
\hline
\end{tabular}

$C A G$, cerebral angiography; Cryo, cryoablation; LAAC, left atrial appendage closure.

NOAC, non-vitamin K antagonist oral anticoagulant; RFCA, radiofrequency ablation; SR, sinus rhythm.

a Time and rhythm were recorded when the event occuOrred.

TABLE 5 | Univariate and multivariate analysis of clinical variables affecting CCE.

\begin{tabular}{|c|c|c|c|c|c|c|c|c|}
\hline & Total no. & $\begin{array}{c}\text { CCE } \\
\text { no. (\%) }\end{array}$ & \multicolumn{2}{|c|}{ Univariate analysis } & \multicolumn{2}{|c|}{ Multivariate analysis (Model 1) } & \multicolumn{2}{|c|}{ Multivariate analysis (Model 2) } \\
\hline Male & 2977 & $151(5.1)$ & $1.297(0.999-1.683)$ & 0.050 & $1.199(0.916-1.569)$ & 0.186 & & \\
\hline Persistent AF & 930 & $52(5.6)$ & 1.009 (0.737-1.382) & 0.954 & $1.084(0.786-1.495)$ & 0.624 & $1.489(1.144-1.938)$ & 0.938 \\
\hline Hypertension & 1799 & $112(6.2)$ & $1.233(0.954-1.594)$ & 0.109 & $1.012(0.774-1.324)$ & 0.929 & & \\
\hline CAD & 699 & $46(6.6)$ & $1.243(0.893-1.729)$ & 0.198 & $1.128(0.803-1.583)$ & 0.488 & & \\
\hline Vascular disease & 507 & $34(6.7)$ & $1.257(0.865-1.828)$ & 0.230 & $1.175(0.801-1.723)$ & 0.411 & & \\
\hline Heart failure & 130 & $10(7.7)$ & $1.435(0.743-2.770)$ & 0.282 & $1.349(0.686-2.650)$ & 0.386 & & \\
\hline Prior stroke/TIA & 290 & $24(8.3)$ & $1.261(1.013-1.571)$ & 0.038 & $1.202(0.960-1.506)$ & 0.109 & & \\
\hline Renal dysfunction & 88 & $12(13.6)$ & $2.770(1.486-5.163)$ & 0.001 & $2.485(1.309-4.718)$ & 0.005 & & \\
\hline $\mathrm{CHA}_{2} \mathrm{DS}_{2}-\mathrm{VASc} \geq 2$ & 1959 & $134(6.8)$ & $1.534(1.188-1.980)$ & 0.001 & & & $1.488(1.143-1.937)$ & 0.003 \\
\hline Uninterrupted VKA & 796 & $38(4.8)$ & $1.758(1.148-2.695)$ & 0.010 & $1.708(1.111-2.624)$ & 0.015 & $1.755(1.144-2.692)$ & 0.010 \\
\hline Bridging heparin ${ }^{b}$ & 1848 & $161(8.7)$ & $3.348(2.432-4.608)$ & $<0.001$ & $3.539(2.564-4.885)$ & $<0.001$ & $3.462(2.511-4.773)$ & $<0.001$ \\
\hline
\end{tabular}

CCE, combined complication endpoint; CAD, coronary artery disease; NOAC, non-vitamin K antagonist oral anticoagulant; TIA, transient ischemic attack; VKA, vitamin K antagonist. a Uninterrupted VKA : uninterrupted VKA vs. minimally interrupted NOAC.

${ }^{b}$ Bridging heparin: bridging heparin vs. minimally interrupted NOAC.

their clinical characteristics (Table 5). Age, male gender, prior stroke/TIA, renal dysfunction, $\mathrm{CHA}_{2} \mathrm{DS}_{2}$-VASc $\geq 2$, HAS-BLED $\geq 3$, and anticoagulation strategies were found to be significantly associated with occurrence of CCE.

Subsequently, a multivariable analysis was performed with the logistic regression model (model 1). With the exception of $\mathrm{CHA}_{2} \mathrm{DS}_{2}$-VASc $\geq 2$ and HAS-BLED $\geq 3$, the significant confounders identified in the univariable test were included in the model. Besides, some other clinically important covariates were added to the model despite their nonsignificant association in the univariate test. After adjustment for these confounders, it was found that the age, renal dysfunction, and anticoagulation strategies were independent risk factors of CCE. To avoid multicollinearity, another multivariable model was run by including $\mathrm{AF}$ type, $\mathrm{CHA}_{2} \mathrm{DS}_{2}$-VASc $\geq 2$, HAS-BLED $\geq 3$, and anticoagulation strategies (model 2). As observed in this model, all the aforementioned confounders were independently associated with CCE except the type of AF. In both models, the minimally interrupted NOAC strategy was considered the reference and was compared with the uninterrupted VKA strategy and bridging heparin strategy. The odds ratios from these two models are presented in Table 5. 
Cumulative event rates of CCE between subgroups within 30 days after the ablation procedure were shown by a KaplanMeier analysis. After adjusted by age, AF type, $\mathrm{CHA}_{2} \mathrm{DS}_{2}$-VASc, and HAS-BLED score, a significant difference of CCE rates were shown in the Minimally interrupted NOAC group as compared with the Uninterrupted VKA group (2.77 vs. $4.77 \%, p=0.008$ )
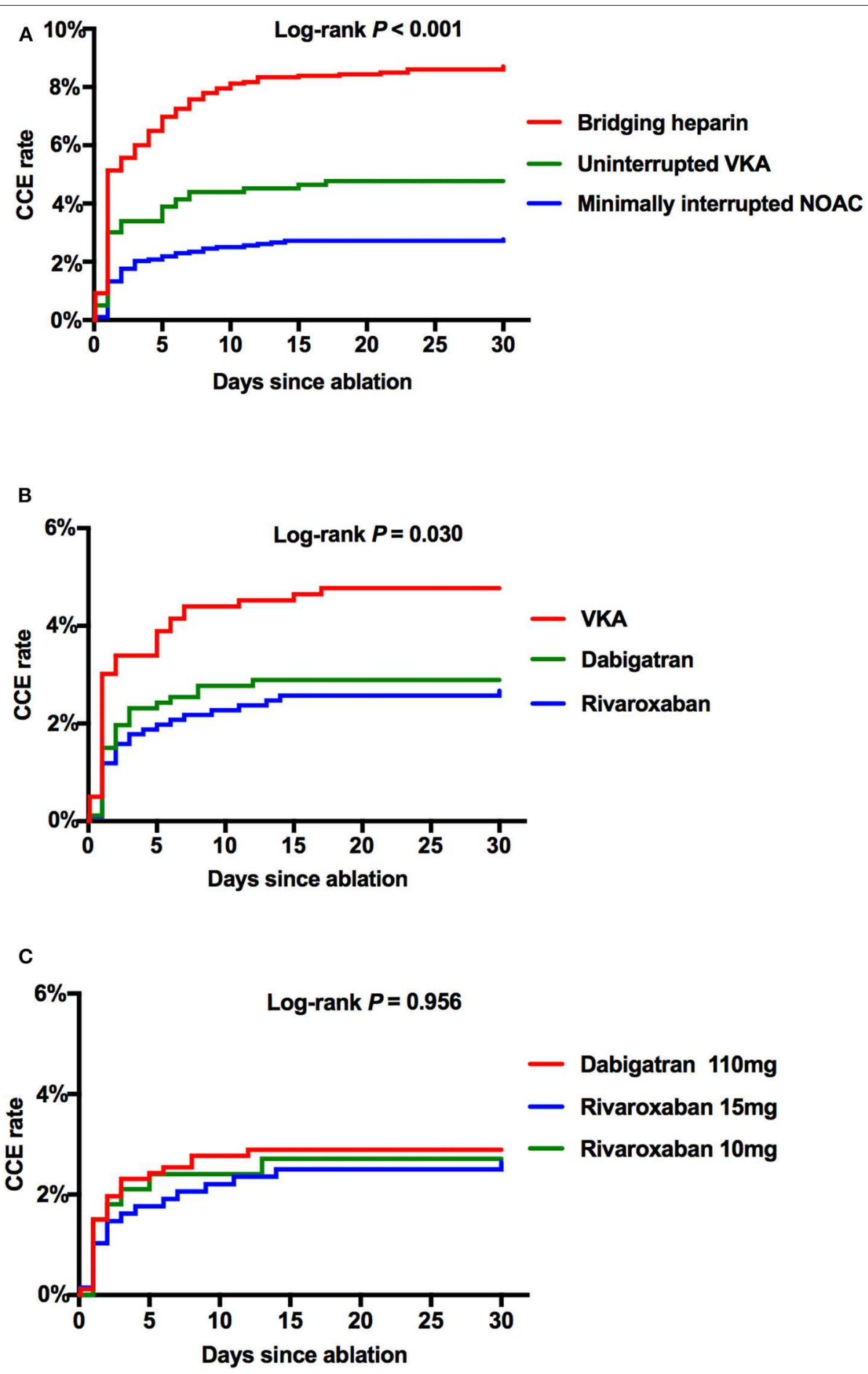

FIGURE 3 | The Kaplan-Meier curves showing the event rates of CCE after the ablation procedure. (A) Comparison among Bridging, VKA, and NOAC. (B) Comparison among VKA, dabigatran $110 \mathrm{mg}$, and total rivaroxaban. (C) Comparison among dabigatran $110 \mathrm{mg}$, rivaroxaban $15 \mathrm{mg}$, and rivaroxaban $10 \mathrm{mg}$. CCE, combined complication endpoint; NOAC, non-vitamin K antagonist oral anticoagulant; VKA, vitamin K antagonist. 
and the Bridging heparin group (2.77 vs. $8.71 \%, p<0.001)$ (Figure 3A). Further subgroup analyses were performed between the VKA group and individual NOAC groups. The incidence rate of CCE was similar between the dabigatran group and the rivaroxaban group ( 2.89 vs. $2.67 \%, p=0.773$ ), and a similar trend was observed for the comparison between the rivaroxaban $15 \mathrm{mg}$ and $10 \mathrm{mg}$ groups (2.65 vs. $2.71 \%, p=0.956$ ) (Figures 3B,C).

\section{DISCUSSION}

We retrospectively evaluated the safety and effectiveness of minimally interrupted NOAC compared with bridging therapy and uninterrupted VKA for nonvalvulare $\mathrm{AF}$ ablation in a real-world setting. Furthermore, NOACs were also compared individually with uninterrupted VKAs in our study. The main findings were as follows: (1) the risk of thromboembolism in the current anticoagulant regimens is exceedingly rare and not significantly different between groups; (2) minimally interrupted NOACs are associated with a lower incidence of bleeding complications in comparison with uninterrupted warfarin and bridging therapy; (3) the incidence of CCE was similar between the dabigatran and rivaroxaban groups.

Great advances have been made in periprocedural anticoagulation therapy for patients with $\mathrm{AF}$ ablation during the past few years. In the warfarin era, continuous warfarin therapy during $\mathrm{AF}$ ablation reduced the risks of thromboembolic and bleeding complications as compared with a bridging regimen with LMWH (2). This uninterrupted warfarin strategy had gained wider acceptance and was endorsed in the 2012 expert consensus statement and 2014 AHA/ACC/HRS guideline $(14,15)$. With the emergence of NOACs, increasing numbers of patients with AF are on NOACs during the catheter ablation due to its advantages of rapid onset of therapeutic anticoagulation, shorter duration of action, and relatively fewer drug interactions. In recent years, several meta-analyses and RCTs favored performing AF ablation with uninterrupted NOACs, as this strategy has been linked to a similar incidence of thromboembolic events and lower incidence of bleeding complications compared to uninterrupted warfarin therapy (3-9). However, an antidote is not commercially available for NOACs (except for dabigatran), and the existing published data about reversal agents from experienced centers are limited. The major concern of uninterrupted NOACs might be the risk of major bleeding during ablation, particularly in Asian populations, who have a higher risk of bleeding than persons of other races (16). Therefore, a minimally interrupted NOAC during $\mathrm{AF}$ ablation as an alternative to uninterrupted NOACs strategy in our center seems to be reasonable, which also gained a IIa indication in the 2017 expert consensus statement (1).

While previous studies had demonstrated less major bleeding with continues NOAC versus VKA, this NOAC dosing strategy does not reflect widespread current clinical practice (9). The European Snapshot Survey on Procedural Routines in Atrial Fibrillation Ablation (ESS-PRAFA) in 2015 demonstrated that only $14 \%$ of patients were on an NOAC uninterrupted therapy (10). However, a majority of the patients (53\%) underwent the procedure with a minimally interrupted NOAC strategy (one or two doses withheld), followed by $34 \%$ of the patients on interrupted NOAC $\geq 2$ days. As compared with the uninterrupted warfarin strategy, the ABRIDGE-J trial showed that minimally interrupted dabigatran (one or two doses withheld) was associated with fewer major bleeding events without compromising thromboembolic safety (17). In addition, results from the Apixaban Evaluation of Interrupted Or Uninterrupted anticoagulation for ablation of atrial fibrillation (AEIOU) randomized trial showed no difference between continuous apixaban and minimally interrupted apixaban (one dose withheld) for the risk of major bleeding and thromboembolic events (18). Furthermore, a recent metaanalysis including 13 studies (4 randomized and 9 observational) with 5463 patients found that minimally interrupted and continuous NOAC strategy were both safe and non-inferior periprocedural strategies in comparison to uninterrupted VKA (19). Given the current lack of standardized protocols for the periprocedural management of minimally interrupted NOAC and interrupted or uninterrupted VKA therapy still a common strategy (20), our study based on a modest proportional shift away from bridging and VKA toward NOAC use over time which reflecting a real-world practice of periprocedural anticoagulant therapy. Furthermore, we found a similar low complication rates in patients receiving dabigatran as compared to rivaroxaban. There was no statistical difference with regard to the incidence rates of CCE between different NOAC groups.

Previous studies reported the incidence of periprocedural thromboembolic events in patients undergoing $\mathrm{AF}$ ablation ranges from 0.1 to $1.1 \%(21,22)$, and bleeding complications were occurred within a range of $12-20 \%$ (23). In our study, the overall rate of thromboembolic events was $0.22 \%$ in the Bridging heparin group, $0.25 \%$ in the Uninterrupted VKA group, and $0.11 \%$ in the Minimally interrupted NOAC group. The overall rate of bleeding complications was $8.50 \%$ in the Bridging heparin group, $4.52 \%$ in the Uninterrupted VKA group, and $2.67 \%$ in the Minimally interrupted NOAC group. This is comparable, albeit lower than in previously studies. Currently, one of the most important reasons to choose an uninterrupted NOAC or VKA strategy instead of a minimally interrupted NOAC therapy is to minimize the incidence of periprocedural thromboembolic events. However, we found a $50 \%$ relative risk reduction in thromboembolic complications with minimally interrupted NOACs $(0.11 \%)$ as compared with uninterrupted VKA $(0.25 \%)$, although the single endpoint missed statistical significance. It should be emphasized that uninterrupted NOAC or VKA does not prevent all acute brain lesion, which can be caused by air embolism or debris from ablation lesions (24). Furthermore, a low event rates of thromboembolism might result in a limited statistical power to delineate differences among groups.

As for major bleeding, a similar low incidence was noted (Minimally interrupted NOAC $0.11 \%$ vs. Uninterrupted VKA $0.50 \%)$ while no significant difference was detected $(p=0.073)$. It should be considered that pericardial tamponade-the only major bleeding events in our study-is mostly caused by technical factors. It is important to note that it is not the anticoagulant that causes spontaneous bleeding, but the inherent 
risk of the procedure. A misdirected transseptal puncture, direct mechanical trauma, and overheating during the radiofrequency energy delivery are the most common causes leading to cardiac tamponade (14). Recently, a study focused on the management of cardiac tamponade in AF ablation had revealed there was no significant difference between the anticoagulant used with the occurrence of events (25), which was consistent with our findings.

Previous meta-analysis reported a reduced incidence of nonmajor bleeding when withholding several does of NOACs before the procedure (8). In our study, patients in the minimally interrupted NOACs group had a lower risk of suffering nonmajor bleeding events both in comparison to the Uninterrupted warfarin group $(p=0.044)$ and the Bridging Heparin group $(p<0.001)$. Patient-related bleeding risk was very low in our study because $97 \%$ of the patients had a HAS-BLED $<3$. As the minor groin complications (including groin bleeding/hematoma, arteriovenous fistula, and pseudoaneurysm) were the most frequent non-major bleeding complications related to the technical factors, the influence of periprocedural anticoagulation on the complications is rather negligible. It should be noted that increasing numbers of patients with AF are on NOACs during the catheter ablation in the past 3 years, while bridging therapy and uninterrupted VKA were mostly used in our center before 2016. With the accumulation of procedure experience, this may lead to a decrease in ablation-related bleeding complications.

Undoubtedly, there are some limitations in our observational study. First, the low incidence of events likely represented a population of highly experienced electrophysiologists performing the catheter ablation, which could have reduced the sensitivity of the results. Second, given that the grouping was retrospective based on medical records, a modest proportional shift away from bridging and VKA toward NOAC use over time was seen. As a result, there was a bias caused by historical changes in periprocedural anticoagulation strategies. Third, no routine MRI was performed to detect asymptomatic cranial lesions following the ablation, which might have underestimated the incidence of stroke. Fourth, this study was conducted in Chinese patients; thus, the findings cannot be generalized to other ethnic populations. Despite these limitations, our study was carried out under the real-world use of periprocedural anticoagulation strategy in AF ablation during the past few years, which might provide some valuable insights into clinical practice.

\section{REFERENCES}

1. Calkins H, Hindricks G, Cappato R, Kim YH, Saad EB, Aguinaga L, et al. 2017 HRS/EHRA/ECAS/APHRS/SOLAECE expert consensus statement on catheter and surgical ablation of atrial fibrillation. Heart Rhythm. (2017) 14:e275-e444. doi: 10.1016/j.hrthm.2017.05.012

2. Di Biase L, Burkhardt JD, Santangeli P, Mohanty P, Sanchez JE, Horton R, et al. Periprocedural stroke and bleeding complications in patients undergoing catheter ablation of atrial fibrillation with different anticoagulation management: results from the role of coumadin in preventing thromboembolism in atrial fibrillation (AF) patients undergoing catheter ablation (COMPARE) randomized trial. Circulation. (2014) 129:2638-44. doi: 10.1161/CIRCULATIONAHA.113.006426

\section{CONCLUSIONS}

In patients undergoing AF ablation, minimally interrupted NOACs during the periprocedural period appears safer and equally effective compared to the bridging heparin and uninterrupted VKA therapy. Both minimally interrupted dabigatran or rivaroxaban (one dose withheld) showed a noninferior and even superior combined complication outcome compared to uninterrupted warfarin during the periprocedural period of the AF ablation.

\section{DATA AVAILABILITY STATEMENT}

The datasets generated for this study are available on request to the corresponding author.

\section{ETHICS STATEMENT}

The studies involving human participants were reviewed and approved by Clinical Research Ethics Committee of Guangdong Provincial People's Hospital. The patients/participants provided their written informed consent to participate in this study. Written informed consent was obtained from the individual(s) for the publication of any potentially identifiable images or data included in this article.

\section{AUTHOR CONTRIBUTIONS}

LT, HaL, HD, SW, and YX contributed conception and design of the study. LT, HaL, XZ, XF, HL, and YL organized the database. LF, ZF, and HuL performed the statistical analysis. LT and HaL wrote the first draft of the manuscript. LT, HaL, $\mathrm{HD}$, and YX wrote sections of the manuscript. All authors contributed to the manuscript revision and read and approved the submitted version.

\section{FUNDING}

This work was supported by the National Key Research and Development Projects (Nos. 2018YFC1312501 and 2018YFC1312502) and the National Natural Science Foundation (No. 81870254). The study funders played no role in the design or in the conduct of this study.

3. Calkins H, Willems S, Gerstenfeld EP, Verma A, Schilling R, Hohnloser $\mathrm{SH}$, et al. Uninterrupted dabigatran versus warfarin for ablation in atrial fibrillation. N Engl J Med. (2017) 376:1627-36. doi: 10.1056/NEJMoa17 01005

4. Cappato R, Marchlinski FE, Hohnloser SH, Naccarelli GV, Xiang J, Wilber DJ, et al. Uninterrupted rivaroxaban vs. uninterrupted vitamin $\mathrm{K}$ antagonists for catheter ablation in non-valvular atrial fibrillation. Eur Heart J. (2015) 36:1805-11. doi: 10.1093/eurheartj/ehv177

5. Kuwahara T, Abe M, Yamaki M, Fujieda H, Abe Y, Hashimoto K, et al. Apixaban versus warfarin for the prevention of periprocedural cerebral thromboembolism in atrial fibrillation ablation: multicenter prospective randomized study. J Cardiovasc Electrophysiol. (2016) 27:549-54. doi: $10.1111 /$ jce. 12928 
6. Hohnloser SH, Camm J, Cappato R, Diener HC, Heidbüchel H, Mont L, et al. Uninterrupted edoxaban vs. vitamin $\mathrm{K}$ antagonists for ablation of atrial fibrillation: the ELIMINATE-AF trial. Eur Heart J. (2019) 40:3013-21. doi: 10.1093/eurheartj/ehz190

7. Wu S, Yang YM, Zhu J, Wan HB, Wang J, Zhang H, et al. Metaanalysis of efficacy and safety of new oral anticoagulants compared with uninterrupted vitamin $\mathrm{k}$ antagonists in patients undergoing catheter ablation for atrial fibrillation. Am J Cardiol. (2016) 117:926-34. doi: 10.1016/j.amjcard.2015.12.027

8. Zhao Y, Yang Y, Tang X, Yu X, Zhang L, Xiao H. New oral anticoagulants compared to warfarin for perioperative anticoagulation in patients undergoing atrial fibrillation catheter ablation: a meta-analysis of continuous or interrupted new oral anticoagulants during ablation compared to interrupted or continuous warfarin. J Interv Card Electrophysiol. (2017) 48:267-82. doi: 10.1007/s10840-016-0221-7

9. Cardoso R, Knijnik L, Bhonsale A, Miller J, Nasi G, Rivera M, et al. An updated meta-analysis of novel oral anticoagulants versus vitamin $\mathrm{K}$ antagonists for uninterrupted anticoagulation in atrial fibrillation catheter ablation. Heart Rhythm. (2018) 15:107-15. doi: 10.1016/j.hrthm.2017.09.011

10. Potpara TS, Larsen TB, Deharo JC, Rossvoll O, Dagres N, Todd D, et al. Oral anticoagulant therapy for stroke prevention in patients with atrial fibrillation undergoing ablation: results from the first european snapshot survey on procedural routines for atrial fibrillation ablation (ESS-PRAFA). Europace. (2015) 17:986-93. doi: 10.1093/europace/euv132

11. Mehran R, Rao SV, Bhatt DL, Gibson CM, Caixeta A, Eikelboom J, et al. Standardized bleeding definitions for cardiovascular clinical trials: a consensus report from the Bleeding Academic Research Consortium. Circulation. (2011) 123:2736-47. doi: 10.1161/CIRCULATIONAHA.110.009449

12. Schulman S, Kearon C. Subcommittee on Control of Anticoagulation of the Scientific and Standardization Committee of the International Society on Thrombosis and Haemostasis. Definition of major bleeding in clinical investigations of antihemostatic medicinal products in non-surgical patients. J Thromb Haemost. (2005) 3:692-4. doi: 10.1111/j.1538-7836.2005.01204.x

13. Beyls C, Hermida A, Duchateau J, Maury P, Taieb J, Laurent G, et al. Management of acute cardiac tamponade by direct autologous blood transfusion in interventional electrophysiology. J Cardiovasc Electrophysiol. (2019) 30:1287-93. doi: 10.1111/jce.14050

14. Calkins H, Kuck KH, Cappato R, Brugada J, Camm AJ, Chen SA, et al. 2012 HRS/EHRA/ECAS Expert Consensus Statement on Catheter and Surgical Ablation of Atrial Fibrillation: recommendations for patient selection, procedural techniques, patient management and follow-up, definitions, endpoints, and research trial design. Europace. (2012) 14:528-606. doi: 10.1093/europace/eus027

15. January CT, Wann LS, Alpert JS, Calkins H, Cigarroa JE, Cleveland JC Jr, et al. 2014 AHA/ACC/HRS guideline for the management of patients with atrial fibrillation: a report of the American College of Cardiology/American Heart Association Task Force on Practice Guidelines and the Heart Rhythm Society. J Am Coll Cardiol. (2014) 64:e1-76. doi: 10.1016/j.jacc.2014.03.022

16. Lip GY, Wang KL, Chiang CE. Non-vitamin $\mathrm{K}$ antagonist oral anticoagulants (NOACs) for stroke prevention in Asian patients with atrial fibrillation: time for a reappraisal. Int J Cardiol. (2015) 180:246-54. doi: 10.1016/j.ijcard.2014.11.182

17. Nogami A, Harada T, Sekiguchi Y, Otani R, Yoshida Y, Yoshida $\mathrm{K}$, et al. Safety and efficacy of minimally interrupted dabigatran vs uninterrupted warfarin therapy in adults undergoing atrial fibrillation catheter ablation: a randomized clinical trial. JAMA Netw Open. (2019) 2:e191994. doi: 10.1001/jamanetworkopen.2019.1994

18. Reynolds MR, Allison JS, Natale A, Weisberg IL, Ellenbogen KA, Richards $\mathrm{M}$, et al. A prospective randomized trial of apixaban dosing during atrial fibrillation Ablation: The AEIOU Trial. JACC Clin Electrophysiol. (2018) 4:580-8. doi: 10.1016/j.jacep.2017.11.005

19. Ha FJ, Barra S, Brown AJ, Begley DA, Grace AA, Agarwal S. Continuous and minimally-interrupted direct oral anticoagulant are both safe compared with vitamin $\mathrm{K}$ antagonist for atrial fibrillation ablation: An updated meta-analysis. Int J Cardiol. (2018) 262:51-6. doi: 10.1016/j.ijcard.2018.03.095

20. Gunawardene M, Willems S, Schäffer B, Moser J, Akbulak RÖ, Jularic $\mathrm{M}$, et al. Influence of periprocedural anticoagulation strategies on complication rate and hospital stay in patients undergoing catheter ablation for persistent atrial fibrillation. Clin Res Cardiol. (2017) 106:38-48. doi: 10.1007/s00392-016-1021-x

21. Takami M, Lehmann HI, Parker KD, Welker KM, Johnson SB, Packer DL. Effect of left atrial ablation process and strategy on microemboli formation during irrigated radiofrequency catheter ablation in an in vivo model. Circ Arrhythm Electrophysiol. (2016) 9:e003226. doi: 10.1161/CIRCEP.115.003226

22. Cappato R, Calkins H, Chen SA, Davies W, Iesaka Y, Kalman J, et al. Updated worldwide survey on the methods, efficacy, and safety of catheter ablation for human atrial fibrillation. Circ Arrhythm Electrophysiol. (2010) 3:32-8. doi: 10.1161/CIRCEP.109.859116

23. Kwak JJ, Pak HN, Jang JK, Kim SK, Park JH, Choi JI, et al. Safety and convenience of continuous warfarin strategy during the periprocedural period in patients who underwent catheter ablation of atrial fibrillation. J Cardiovasc Electrophysiol. (2010) 21:620-5. doi: 10.1111/j.1540-8167.2009.01670.x

24. Takahashi A, Kuwahara T, Takahashi Y. Complications in the catheter ablation of atrial fibrillation: incidence and management. Circ J. (2009) 73:221-6. doi: 10.1253/circj.cj-08-1097

25. Hamaya R, Miyazaki S, Taniguchi H, Kusa S, Nakamura H, Hachiya H, et al. Management of cardiac tamponade in catheter ablation of atrial fibrillation: single-centre 15 year experience on 5222 procedures. Europace. (2018) 20:1776-82. doi: 10.1093/europace/eux307

Conflict of Interest: The authors declare that the research was conducted in the absence of any commercial or financial relationships that could be construed as a potential conflict of interest.

Copyright (C) 2020 Tang, Liu, Deng, Zhan, Fang, Liao, Liu, Fu, Fu, Liu, Wu and Xue. This is an open-access article distributed under the terms of the Creative Commons Attribution License (CC BY). The use, distribution or reproduction in other forums is permitted, provided the original author(s) and the copyright owner(s) are credited and that the original publication in this journal is cited, in accordance with accepted academic practice. No use, distribution or reproduction is permitted which does not comply with these terms. 